\title{
Description of Topography of Surfaces and Thin Films with the use Fourier Transformation, Obtained from Non-Standard Optical Measurements
}

\author{
Janusz Jaglarz \\ Institute of Physics, Cracow University of Technology \\ Poland
}

\section{Introduction}

This chapter explains how to interpret the results obtained from optical scattering study to characterize topography surfaces and films. In particular, it focuses on measurement of bidirectional reflectance distribution function (BRDF) and on optical profilometry (PO). These techniques allow to determine two main functions describing surface topography, namely: power spectral density (PSD) and autocorrelation function (ACF). They characterize any real surface quantitatively and qualitatively. The PSD and ACF functions are commonly calculated from Fourier transform (FT) of the surface profiles determined in AFM, SEM or PO) measurements.

The optical scattering is directly related to material and surface properties. We can distinguish three types of scatterings base on their features: 1) Topographic scattering resulting fom roughness causing phase fluctuations impressed on the reflected wavefront by the surface height variation [Beckmann and Spizinochino 1963] 2) material scattering created by fluctuations in the composition or density of the surface material [Elson 1984]. Defect scattering is resulting from a presence of sparse distribution of some surface features responsible for scattering, is distributed broadly and continuously over surfaces such as pits or bumps in case of topography and patches of different reflectivity in the case of material scattering [Stover 1995a].

One should emphasize that the roughness is the main source of scattering on surfaces at visible wavelengths range.

Topography of real surface may be described using some fundamental parameters described in national and international norms [ANSI/ ASME B46.1, ISO 2517]

For macroscopic characterization of surface the following terms is applied [Whitehause 2003]): 1) Root mean square roughness $\sigma$ (rms). It is calculated by the vertical deviations of a real surface from its ideal form. 2) The rms slope $s$ and correlation length which is expressed by the ratio: $T=2^{1 / 2} \sigma / s$. 3) The power coefficient the type of qualifying statistical distribution of surface heights. The distance between respective surface features is defined as spatial wavelengths. In topographic analysis the term spatial frequency which is a reverse of spatial wavelength is used. 
The given statistical terms are calculated from the surface-profile data in a specified spatial bandwidths. The range of measured spatial wavelengths is determined by lateral resolution of measuring tool and sampling length in short and long wavelength limit respectively.

\section{The power spectral density and autocorrelation function}

The real surface and films are described by two statistical functions, namely autocorrelation (ACF) and power spectral density (PSD) functions [Bennett and Mattsson 1999]. PSD function is defined in spatial frequency domain and it expresses the roughness power per unit frequency over the sampling length. From mathematical point of view, PSD is evaluated from Fourier transform (FT) of surface profile $h(r)$. For one dimensional profile $h(r)$ PSD is described as:

$$
\operatorname{PSD}\left(f_{r}\right)=\lim _{L \rightarrow \infty}\left\langle\left|\frac{2}{L} \int_{-L / 2}^{L / 2} h(r) e^{i 2 \pi f} d r\right|^{2}\right\rangle
$$

Where $f$-is spatial frequency and $L$ is sampling length. The units one dimensional PSD are length to third power.

The roughness and average slopes are first and second statistical momentums of PSD function so they may well be directly calculated from PSD frequency spectra by the following integrals:

$$
\begin{gathered}
\sigma^{2}=\int_{f_{\min }}^{f_{\max }} P S D(f) d f \\
s^{2}=\int_{f_{\min }}^{f_{\max }}(2 \pi f)^{2} P S D(f) d f
\end{gathered}
$$

If values of PSD are known, one can determine the statistical parameters, such as root-mean square (rms) roughness, slopes and correlation length by using $\mathrm{ABC}$ model which describe PSD in simple analytical formula [Elson and Benett 1995]:

$$
P S D(f)=A\left[1+(B f)^{2}\right]^{-C / 2}
$$

where $A, B, C$ are model parameters related with basic quantities characterizing a surface.

The $A$ parameter is a $P S D(f)$ value for low frequency formula, $B / 2 \pi$ is correlation length and $C$ determine type of power law in high spatial frequency. The $A B C$ model applies for single surface.

The other way of surface characterization is describing it by means of autocorrelation function $A C F(r)$ defined in spatial wavelength domain. Generally the autocorrelation function of surface is used to compare data sets of heigths $h(r)$ to a translated version of itself and the averaging.

$$
A C F(r)=\lim _{L \rightarrow \infty} \frac{1}{L} \int_{-L / 2}^{L / 2} h(r) h(r+\tau) d r
$$


Where translation $\tau$ is so called lag distance. The ACF function is usually calculated from surface profiles obtained in AFM and SEM studies. For many random surfaces the ACF $(r)$ may be expressed in the following form [Bendat and Piersol 1971, Whitehead 2003]:

$$
A C F(r)=A C F(0) \exp \left(-\left|\frac{r}{T}\right|^{2 \alpha}\right)
$$

Where $\operatorname{ACF}(0)$ is the value of autocorrelation function in point $r=0$. The value of coefficient specifies a type of statistical distribution of surface irregularities. So for $\alpha=1 / 2$ and $\alpha=1$ the $\mathrm{ACF}(\mathrm{r})$ describes Lorentzian and Gaussian distribution respectively. For distance $r=T$ the $A C F(r)$ decreases e - fold. Thus the value of $r=T$ defines autocorrelation length. Such defined ACF is equal statistically averaged surface spatial wavelength.

In some surface investigations obtained by the use of optical methods, as in optical profilometry, the following useful relation between average slope $s$ and ACF function could be applied [Stover et. al 1984]:

$$
m^{2}=\lim _{\tau \rightarrow \infty} \frac{d^{2} A C F(\tau)}{d \tau^{2}}
$$

According to Parseval's theorem PSD and ACF provide the same information about surface statistic expressed in space frequency and wavelength domain.

Therefore the PSD and ACF may express by using Wiener - Kninchin relations [Bendat and Piersol 1986] as Fourier transform and inverse FT:

$$
\begin{gathered}
A C F(r)=\int_{-\infty}^{\infty} \operatorname{PSD}\left(f_{x}\right) e^{j 2 \pi f \tau} d f \\
P S D(f)=\int_{-\infty}^{\infty} A C F(\tau) e^{-j 2 \pi f \tau} d \tau
\end{gathered}
$$

The calculating procedure of macroscopic surface parameters is shown on Fig. 1.

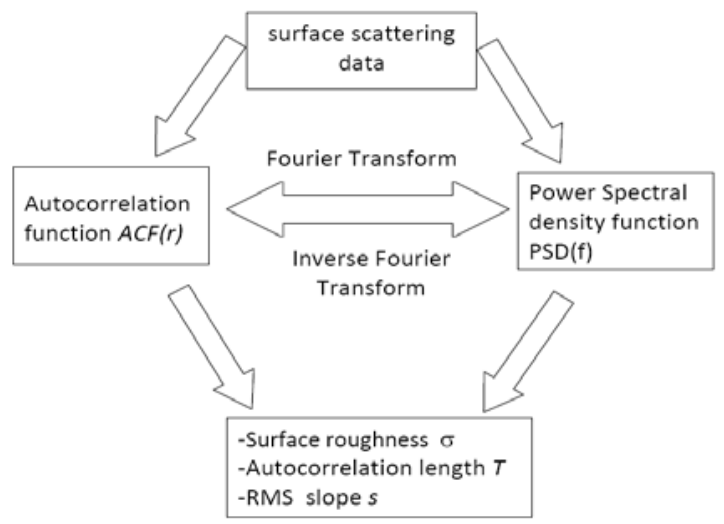

Fig. 1. Scheme of calculating surface parameters procedure from optical measurements 


\section{The BRDF technique}

Optical measurements allow to find macroscopic parameters of surfaces and films from angular measurements of scattered light. The very good tool for single surface and multilayered films characterization is bidirectional reflection function method (BRDF) [Nicodemus 1965].

In BRDF the differential power of scattered beam $d P$ per solid angle of receiver aperture $d \Omega$ in the $\theta$ s direction and per incident power $P_{i}$ coming from the $\theta_{1}$ direction is measured. In Fig. 2a the geometry of incident and measured reflected radian beams have been presented.

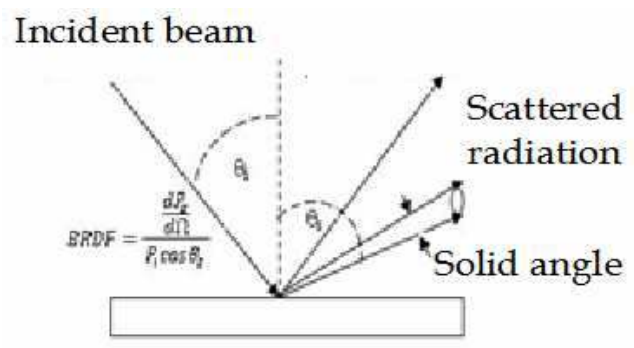

a)

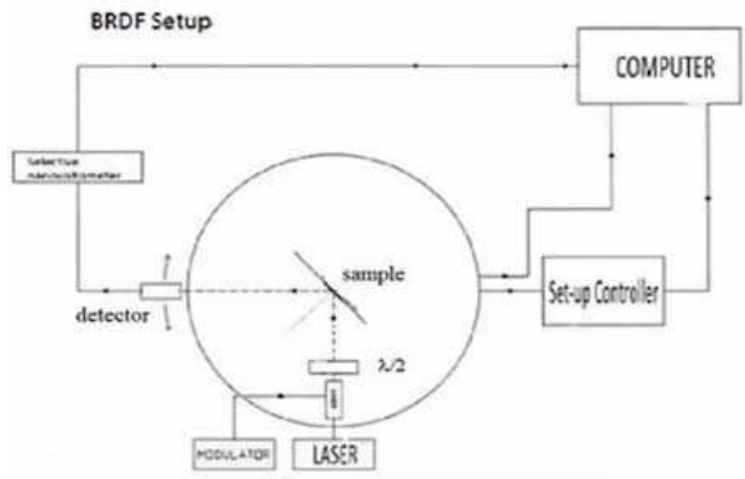

b)

Fig. 2. Geometry of BRDF measurement (a) and scheme of BRDF measurement setup (b)

Practically $d P / d \Omega$ is equal to the measured scatter power $P_{s}$ per acceptance angle $\Omega$ of a detector namely:

$$
B R D F=\frac{d P / d \Omega}{P_{i} \cos \theta_{s}}=\frac{P_{S}}{P_{i} \Omega \cos \theta_{S}}\left[s r^{-1}\right]
$$

If studied surface is relatively flat, the excellent approach give vector The Raleigh-Rice vector perturbation theory [Elson and Benett 1979] is best applied. It shows simple dependence between scattered radiation expressed by BRDF and PSD of investigated surface.

$$
B R D F=\frac{16 \pi^{2}}{\lambda^{2}} \cos \theta_{i} \cos \theta_{s} Q \operatorname{PSD}(f)
$$


where $Q$ is a factor dependent on polarization state of the light source, the optical constants and $\lambda$ where $\lambda$ is the light wavelength. PSD is represented in spatial frequency $f$ that is related with angles $\theta_{i}$ and $\theta_{s}$ by following formula:

$$
f=\frac{\sin \left(\theta_{s}\right)-\sin \left(\theta_{i}\right)}{\lambda}
$$

If the surface roughness is spatially isotropic, as is usually assumed for randomly polished surfaces, the PSD depends only on the magnitude of the surface spatial frequency and is independent of its direction on the surface plane. All information about the surface spectrum can be obtained from scattering measurements made in the plane of incidence. This allows to extract the topographic structure of the single surface from scattering phenomena, which means that BRDF and PSD (except for the factor $Q \cos \theta_{s}$ ) are directly proportional. The so called "golden rule" is a principle of surface investigations by the use angular scatterometry [Church et al. 1977, Stover 1995b].

BRDF measurements presented in this chapter were performed with an automatic scatterometer (Fig. 2b). It consists of a $650 \mathrm{~nm}$ laser diode as the light source (with the beam diameter of $2 \mathrm{~mm}$ ) mounted on a goniometric table with $0.01 \mathrm{deg}$ resolution. The light scattered at the sample surface is measured with a Si photodiode detector. The rotations are obtained by a computer controlled stepper motors. For a fixed angle of incidence, the scattered intensity in the plane of incidence are measured by varying the detector orientation. All measurements are carried out with the s-polarized incident beam. In any case, the sample surface size was much larger than the beam diameter. Moreover, the minimal illuminated area $\left(4 \mathrm{~mm}^{2}\right)$ is large enough to yield meaningful statistical description of the surface [Stover 1984].

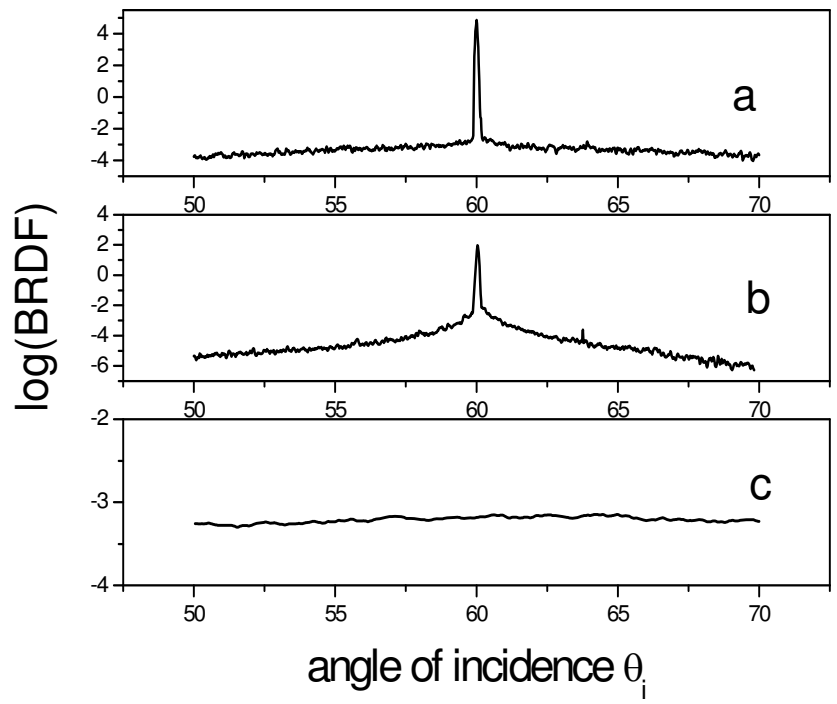

Fig. 3. BRDF vs. angle of incidence for 3 different samples: flat silicon-a, polished steel - $b$, SWM7 and Spectralon - c surfaces 
Fig. 3 shows the results of BRDF measurements as a function of scattering angle for 3 samples. The image in Fig. 3 consists of polished silicon with roughness $\sigma=1 \mathrm{~nm}$ (Fig. 3a), mechanically polished steel $\sigma=69 \mathrm{~nm}$ SWM7 with (Fig. 3b) and lambertian white diffuser Spectralon (Fig. 3c). Because scattered intensity strongly depends on scattering angle and intensity ratio for near specular to far specular scattering may reach several orders so generally the BRDF is shown in logarithmic scale. The incidence angle was the same $\theta_{i}=60^{\circ}$ for all samples. As it is easy to notice the angular BRDF's are different. The BRDF of polished Si as other good mirrors has high narrow specular peak. Outside specular reflection mirror reflectors scatter diffusely light at very low level. Polished steel with moderately high roughness has BRDF with lower and wider specular peak and intensity of scattered light monotonically decrease in larger scattering angle. In Spectralon [Workmann 1998] BRDF specular reflection disappears and light is scattered diffusively and homogeneously in all direction.

In the case of polished surfaces the power spectrum of the surface errors is generally a smooth and broad function of spatial frequency, and the smoothed value of the BRDF is independent of the system pupil function.

In a situation when optical constants are unknown, the polarization factor $Q_{s}$ for " $s$ " polarization could be found from angle dependence of specular reflection coefficient. It is given by the geometric mean of the sample specular reflectances $R\left(\theta_{i}\right)$ and $R\left(\theta_{s}\right)$ at angles $\theta_{i}$ and $\theta_{s}$. For scattered radiation measured in plane of incident $Q_{s}$ is equal [Church et al. 1989, Stover 1995b]:

$$
Q_{s}=\left\{R_{s}\left(\theta_{i}\right) R_{s}\left(\theta_{s}\right)\right\}^{\frac{1}{2}}
$$

This equation allows to determine $Q_{s}$ without knowing the optical constants of the sample. Because $Q_{s}$ is a smooth function, a good curve fit may be found by measuring sample reflectance for several angles of incidence. Of course, if optical indices are known, the $Q_{s}$ factor can be calculated directly from the definition.

Fig. 4 Shows $P S D(f)$ calculated from BRDF. The polarisation factor $Q_{s}$ was determined from specular reflectance measurements performed for 7 incidence angles $\theta_{i}$ from range $60^{\circ}$ to $81^{0}$. The ABC model was fitted to experimental data.

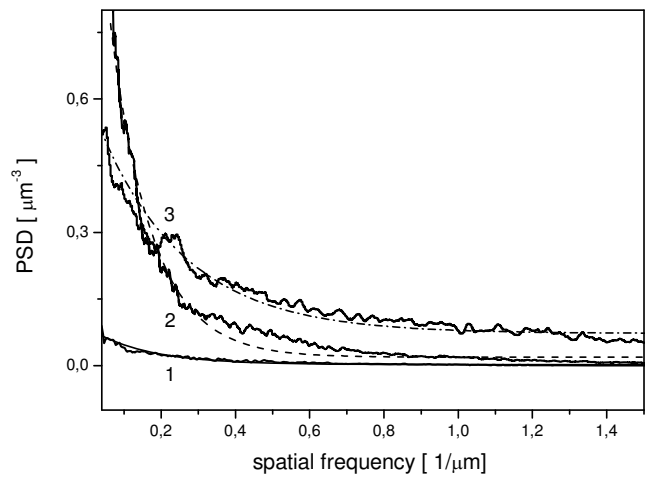

Fig. 4. PSD function extracted from BRDF measurements for TiN films on different substrates on polished: $1-\mathrm{Si}, 2-\mathrm{SWM} 7$ steel and $3-$ on WC plate respectively 
Roughness $\sigma$ of TiN films from equation (4) and autocorrelation length $T$, and power factor $C$ from $A B C$ model (5) have been found. The values of determined parameters are shown in Table 1 . The result strongly suggest the influence of substrate type on topography of TiN films obtained under the same technological conditions.

Log-Log analysis of $P S D(f)$ is very convenient and easy to interpretation of $P S D(f)$ dependence. Many optical surfaces are described by the means ABC model. On Fig. 5 the $\log \log P S D(f)$ for Duralcan alloy before and after $\mathrm{CO}_{2}$ laser annealing [Kaczmar et al. 2000] has been presented.

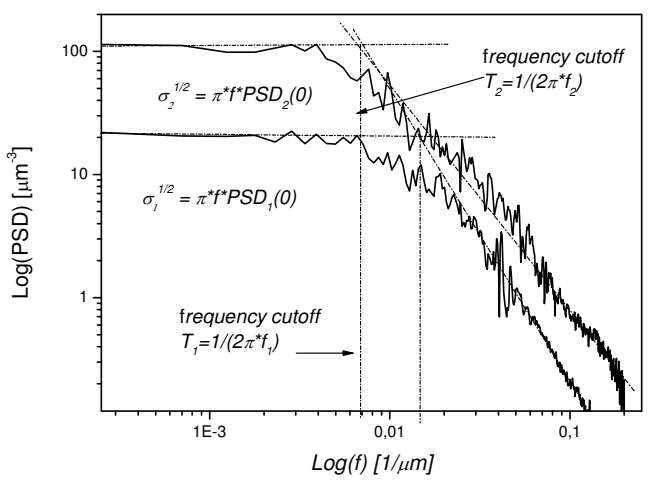

Fig. 5. The values of topographic parameters have been obtained as following:

For low spatial frequencies $\mathrm{Bf}<<1$ the $\operatorname{PSD}(f)$ has constant value. For high frequencies $\mathrm{Bf}>>$ 1 and the formula (3) may be approached by the use $A B C$ model as follows:

$$
\log (P S D)=\log (A)-C \log (B f)
$$

Substituting for $y=\log (P S D)$ and for $x=\log (B f)$, we obtain linear dependence: $y=-C x+b$, where $C$ qualify type of random distribution of irregularietes. For the special case $C=2$ or $C=4$, the distribution of surface highs is Lorentzian or Gaussian respectively.

If we try to extrapolate $P S D(f)$ curves in low and high spatial frequency bandwidth by straight lines one then we will obtain break point on cut of them. It allows to determine from this point so called cut-off frequency. By knowing its value one may to calculated autocorrelation length according formula: $T=1 / 2 \pi^{*} f_{c}$. According to (2) the surface roughness is equal field under the PSD curve. For low frequency bandwidth i.e from zero to cut-off frequency $f_{c}$ one may estimate the $\sigma$ follows to approached formula: $\sigma^{1 / 2}=\pi^{*} f_{c}^{*} P S D(0)$, where $P S D(0)$ denote power spectral density at low frequency.

For some surfaces surfaces in their PSD or BRDF versus frequency the break point do not occur (that is $\mathrm{Bf}>>1$ ). In that case the PSD and BRD may be approximated linear with slope $C$. For random one dimensional surface from $A B C$ model we obtain from (4):

$$
\operatorname{PSD}(f)=A B f^{-C}
$$

The formula (14) expressed in logaritmic terms is the same as (12). Surfaces without break point are called fractal surface [Church 1988]. The autocorrelation length $T$ of fractal profile 
is equal to the length of investigated surface. On Fig. 6 the BRDF versus spatial frequency for 3 fractal surfaces: 1-glass BK7, 2-polished stainless steel, $3-\mathrm{BaSO}_{4}$ white standard is shown.

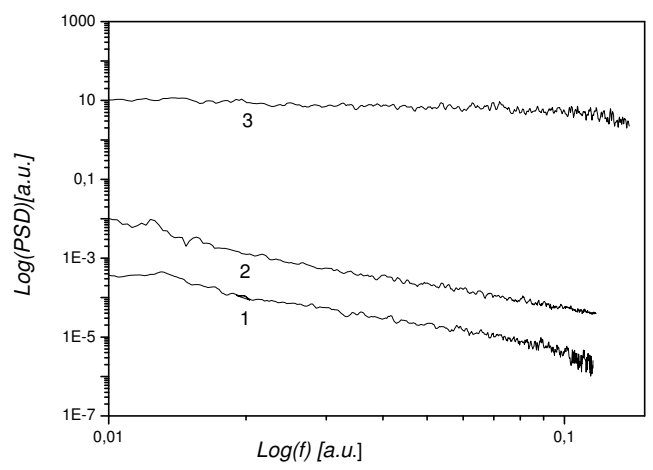

Fig. 6. The power spectral density for fractal profile, 1-BK7 glass, 2-stainless steel, 3-BaSO4 The curves 1 and 2 represents fractal glass and steel surfaces. The $C$ coefficients of power spectrum slopes are nearly 4, so their highs of fractal surface have Gaussian distribution. Curve 3 presents power spectrum for barium sulphate which is applied as Lambertian diffuser [Workmann 1998]. As may easy conclude white standard diffuser are fractal surfaces with zero slope of PSD spectra.

The many practical surfaces in optics or semiconductor application exhibit the fractal-like power spectral density over bandwidths of interest.

As it is known the roughness value depends on spatial bandwidth which is specified by measurements setup and other conditions such as sample size etc. Because PSD spectra of measured surfaces may differ considerably results of roughness may be influenced by position and width of chosen bandwidth. Fig. 7 presents the calculated roughness versus spatial frequency area for SWM7 steel, stainless steel and silicon surfaces respectively.

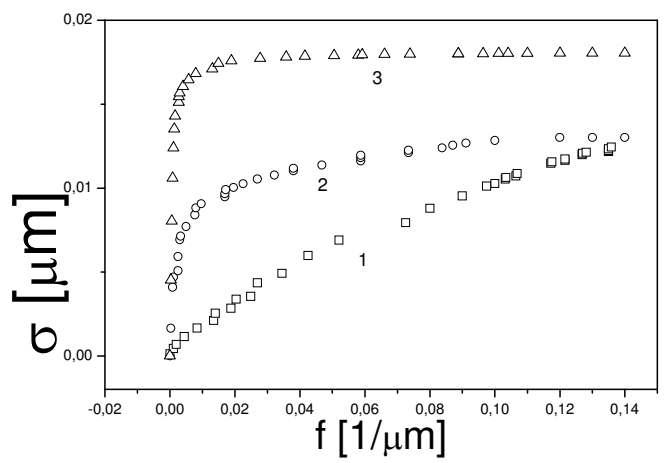

Fig. 7. Surface roughness as a function of spatial frequency bandwidth obtained from BRDF for, SWM7 steel, stainless steel and silicon surface - curve 1, 2, 3 respectively 
As it may be concluded from the above figure, for some surfaces roughness values reach constant value at low frequencies and topographic features observed at high frequencies essentially do not contribute to total roughness.

If the surface roughness contains periodic components, such as tool marks in precisionmachine surfaces, the BRDF will contain sharp diffraction lines. The positions and intensities of those lines are related to the feed rates and amplitudes of the tool marks, while their widths are determined by the system pupil function. The case of periodic surface is shown on Fig. 8. The BRDF for ST3SY steel concurrent (curve 2) and reverse concurrent (curve 1) in milling process is shown.

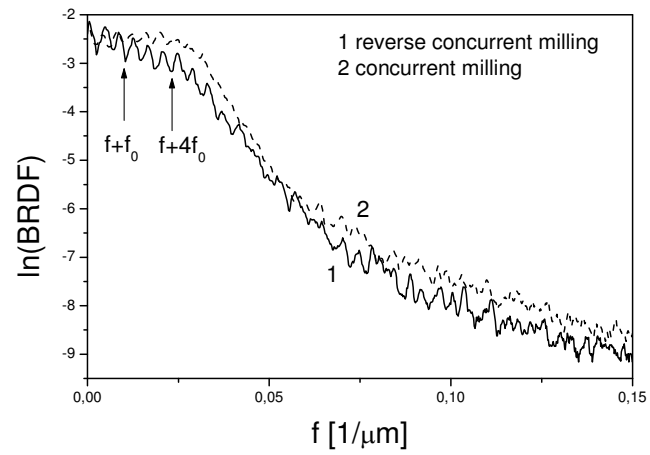

Fig. 8. BRDF for periodic steel surface obtained in milling process

Spatial wavelength of fundamental mill calculated from BRDF is equal $\Lambda_{0}=154 \mu \mathrm{m}$. All remaining peaks from low frequency bandwidth occur on BRDF spectrum in the same interval frequency $f_{0}=1 / \Lambda_{0}$.

Some random optical surface may not be described by the use ABC model. It is for monocrystalline silicon wafer with (100) orientation etched in $\mathrm{KOH}$ solution. In this case reduction of surface reflectivity is a key factor for improving the crystalline silicon solar cell efficiency. In Fig. 9 the relative BRDF frequency spectra for that wafer have been shown.

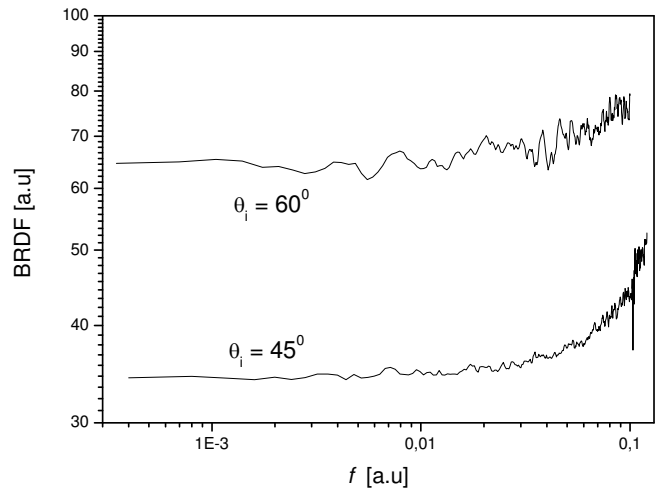

Fig. 9. BRDF of texturized monocrystalline $\mathrm{Si}$ in $\mathrm{KOH}$ for two angles of incidence 
For high spatial frequency slopes have positive values unlike to most optical surfaces. This peculiar behaviour in $B R D F(f)$ dependce is due to surface texture of etched silicon wafer. It consists of pyramid-like irregularietes differing in sizes and shapes [Lipiński and Cichoszewski 2008]. So created texture scatters light into hight angles (frequencies). It allows to substantially reduce reflection for near specular angles.

Additionally one influences of non-topographic features on power spectral density function should be discussed. Such cases can be found in composite (alloys) structures. If components are different the large variations in optical constants may occur. Apart from large reflectance differences, the phase change of reflection for absorbing materials appear. This phase change resulted from reflectance and may equal several tens of degrees for conductors and can be interpret as pseudo-height differences [Bennet and Mattsson 1999]. In other words a hypothetical, perfectly smooth surface made up of elements having different optical constants may show shadowing effect ordinarily interpreted as height differences in optical measurements. For flat surfaces dielectric function variation plays key role in light scattering. This situation can be found in optical study of Al-Si-SiC composites [Kaczmar el al. 2000]. Additionally in Al-Si-SiC structures such a metal-semiconductor-dielectric composites particular monocrystals may occurr on the surface and may be considerably larger than light wavelength. They have own surface direction and reflect light according to geometrical law of reflection.

The phase changes of reflected light which are components of the investigated composites (alloys) result from appreciable differences in their optical constants. In experimental data analysis, the differences in $n$ and $k$ indices particular components could be treated as pseudo-height caused by phase differences. For example for the aboveAl-Si-SiC composites for light with wavelength $\lambda=550 \mathrm{~nm}$, the pseudo-heights are for Al $-24 \mathrm{~nm}, 2.5 \mathrm{~nm}$ for Si and $0 \mathrm{~nm}$ for $\mathrm{SiC}$ for which is dielectric material and was treated as reference. Therefore the pseudo-heights must be taken into consideration when predicting values of power spectral density and autocorreation functions are obtained by the use of optical methods.

\section{The Power spectral density of thin films from BRDF study}

The BRDF results for film depend on topographies of its upper and lower side and in the case of diffusive layers depend also on light scattering in the bulk.

1. If the topography of both upper and bottom interfaces are identical the PSD is given by [Elson 1992]:

$$
B R D F=\left|F_{1}+F_{2}\right|^{2} P S D
$$

where $F_{1}$ and $F_{2}$ are optical function of interfaces film- air and film - substrate respectively, and PSD represents power spectral density function of film system.

2. In the case completely uncorrelated surfaces the BRDF function should be expressed by the formula:

$$
B R D F=\left|F_{1}\right|^{2} P S D_{1}+\left|F_{2}\right|^{2} P S D_{2}
$$

where $P S D_{1}$ and $P S D_{2}$ denote power spectral density for top and bottom film surface respectively. In this case, the scattered power from the different interfaces is added. Anyway one can extract part of scattering coming only from the upper surface using full internal reflection effect in the film. 
Fig 10. shows reflection from transparent film on rough substrate. Part of the light reflect from the top surface, part after refraction on the boundary of the media traverse the film twice due to reflection from substrate and goes out from the sample (see Fig. 10). This situation occurs for specular reflection for any angle of incidence. If however bottom surface is rough, the part radiation is scatter into angles $\theta$ s that are larger than Brewster angle $\theta_{\mathrm{B}}$. For this case the all radiation scattered at angles $\theta \mathrm{s}>\theta_{\mathrm{B}}$ is internally reflected.

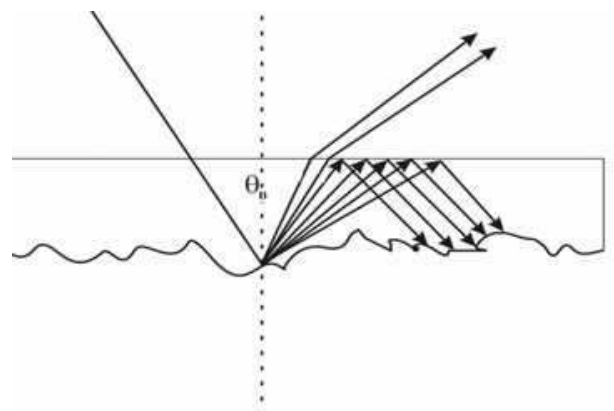

Fig. 10. Propagation of scattered light in layer with rough bottom

It means that the reflected light at suitably great angles comes only from scattering from upper interface of the film and allows to determine PSD from BRDF measurements for upper surface of the film.

If a bulk scattering does not occur the optical losses originate from surface and interface only. In that case determining power spectral density can be done from BRDF measurements. Fig. 11 shows determined PSD function for bare polished silicon 1 - curve 1, thermally obtained $\mathrm{SiO}_{2}$ on the same $\mathrm{Si}$ substrate - curve 2, porous silica and titania-silica blend films on BK7 glass obtained by sol-gel technique [Karasiński 2005] -curve 2 and 3 respectively.

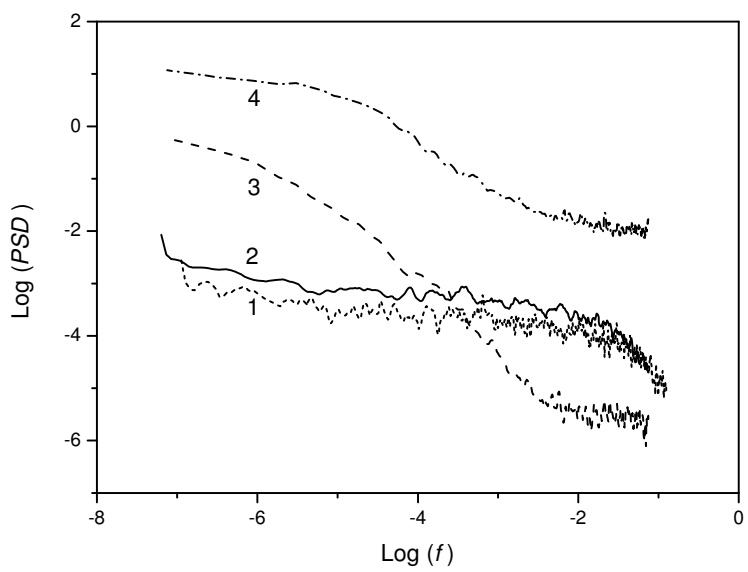

Fig. 11. PSD function of thin films. Curve 1 - bare silicon, 2 -Thermal $\mathrm{SiO}_{2}$ on $\mathrm{Si}, 3$ - porous silica and 4 porous $\mathrm{SiO}_{2}-\mathrm{TiO}_{2}$ on $\mathrm{BK} 7$ glass 
As it may be noticed on Fig. 11 for films 2 and 3 the irregularities with low spatial frequency (longer spatial wavelength) contribute larger fraction to total roughness than the higher ones. Therefore autocorrelation lengths $T$ for layers 1 and 2 are bigger than for thermally obtained silica. The PSD obtained for silicon and $\mathrm{SiO}_{2}$ on the same Si before after annealing are very similar. It results from the fact that upper and lower interfaces of $\mathrm{SiO}_{2}$ film are identical. The values of $\sigma$ determined from AFM and BRDF measurements are similar however roughness calculated from BRDF study are larger. As a matter of fact in BRDF we measure scattered radiation from much bigger surface area than in AFM. The total roughness enlarge due to fraction of irregularities coming from longer spatial wavelengths measured by the use BRDF technique.

The values of macroscopic parameters for surface and films presented in this work have been shown in Table 1. The results given in Table 1 concern optical surfaces. Such surfaces can meet I optics and optoelectronics applications. For those surfaces roughness is commonly less than $5 \mathrm{~nm}$ and their autocorrelation length are order hundred manometers to micrometers. So well average slopes are less than $10^{-2}$. In optics surfaces with slopes less than $10^{-2}$ are regarded as flat [Ohlidal 1988].

\begin{tabular}{|l|c|c|c|c|}
\hline \multicolumn{1}{|c|}{ sample } & $\begin{array}{c}\text { Thickness } \\
{[\mathrm{nm}]}\end{array}$ & $\begin{array}{c}\text { Roughness } \sigma \\
{[\mathrm{nm}]}\end{array}$ & $\begin{array}{c}\mathrm{T} \\
{[\mathrm{nm}]}\end{array}$ & $\mathrm{C}$ \\
\hline Polished $\mathrm{Si}$ & - & 1.7 & 2730 & 3.91 \\
\hline Thermal SiO2 on Si & 352 & 2.9 & 2890 & 3.88 \\
\hline Porous silica on $\mathrm{Si}$ & 672 & 2.1 & 770 & 1.97 \\
\hline titania-silica on $\mathrm{Si}$ & 211 & 1.2 & 455 & 2.11 \\
\hline TiN on Si & 330 & 11.7 & 6780 & 3.61 \\
\hline TiN on steel & 393 & 34 & 13890 & 3.81 \\
\hline Polished aluminium & & 7.3 & 3100 & 3.64 \\
\hline
\end{tabular}

Table 1. The values of topographic parameters of random layers and surfaces

\section{Determination of the PSD and ACF function from optical profilometry}

Measurements of optical reflectance by means of classical reflectometry inform us about optical properties on a large area, i.e. of the order of $1-5 \mathrm{~cm}^{2}$. The results obtained on a much smaller will be similar if coatings and surfaces are homogenous over the investigated area and inside the layers. For inhomogeneous surfaces, when topographic or materials nonuniformities differ from tens $\mu \mathrm{m}$ to several $\mathrm{mm}$, the measurements taken from the integrating sphere measurement and standard reflectometry give rather an averaged reflectance over a larger scale reflected samples.

The scattered radiation measured by optical profilometry (OP) is a function of heights of irregularities and slopes of microfacets, but sensitivity of this method derives mainly from detection of the slope change [Brown and Breitmeier 1988, Whitehead 2003]. The presence of 
long lateral irregularities is often caused by manual or mechanical treatments and may have a periodical nature. The short spatial waves result rather from random process of the surface formation and their contribution to the total profile is easy to determine from atomic force microscopy (AFM) technique.

Optical profilometry measures intensity of reflected radiation from the surface point. Resolution of OP depends on beam diameter where diameters changes from $1 \mu \mathrm{m}$ to $1 \mathrm{~mm}$. In this chapter we presented result of profilometric studies. The long spatial wavelength irregularities detected in OP investigations may contribute substantially to the total roughness. Optical profilometry measurements complete the topography description in long spatial wavelengths.

The optical profilometer described in this chapter is multifunctional experimental set up for surface topography investigations. It work in two modes. The first mode - specular mode employs with laser $\mathrm{He}-\mathrm{Ne}$ as the light source with collimating system allowing to achieve light beam with $12 \mu \mathrm{m}$ diameter. OP measurements have been normalized with the calibration sphere method [Mainsah et al. 1996]. A lead screw stepper motor actuated device may scan $30 \mathrm{~mm} \times 30 \mathrm{~mm}$ surface with step of $0.02 \mathrm{~mm}$. It allows one to obtain the optical profiles of surface with $20 \mu \mathrm{m}$ lateral resolution. The scheme of optical profilometer $(\mathrm{OP})$ is shown in Fig. 12.

The second mode of OP - BRDF mode which is more preferred for rougher films surfaces is based on reflection probe R-200-7. This mode is used to get reflectance over smaller areas at different angles of incidence. The probe consists a bundle of 7 optical fibres, i.e. 6 fibres around one "illumination fibre" (which illuminates sample), each with a diameter of 200 $\mu \mathrm{m}$. Of course the size of illumination defines lateral resolution of measurements. The laser diode $(\lambda=635 \mathrm{~nm})$ is used as the light source. The reflected light is collected by six fibres around the illumination fibre. The probe is coupled with a collecting fibres to a A/D converter connected with computer. The axis of the probe may be angled at $15^{\circ}, 30^{\circ}$ and normal to the sample plane.

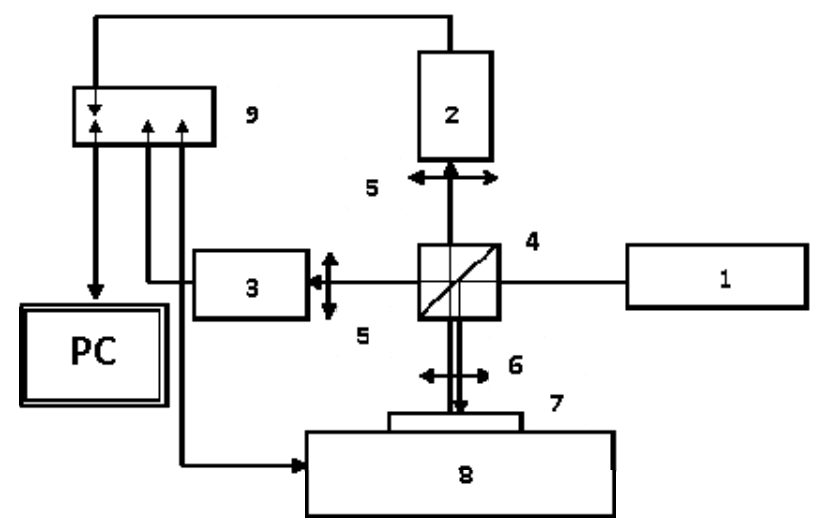

Fig. 12. Scheme of optical profilometer: 1 - laser diode $(\lambda=635 \mathrm{~nm}), 2,3$ - detectors, 4 - beam splitter, 5 - colleting lens, 6 - objective, 7 - sample, 8 -XY stage, 9 - controlling/collecting unit

The analysis of two-dimensional Fourier transforms of surface profiles expressed as PSD function of $f_{x}$ and $f_{y}$ coordinates is an effective tool for any texture characterization. Fourier 
transformations of surface profiles are determinate by the use of fast Fourier transform (FFT) procedure. images allow to find distribution of surface heights in inverse space, distinct their periodicity and evaluate anisotropy of surface films in.

In Fig. $13 \mathrm{a}$ and $\mathrm{b}$, the mechanically polished steel SWM7 block profile, and its twodimensional FFT is presented.

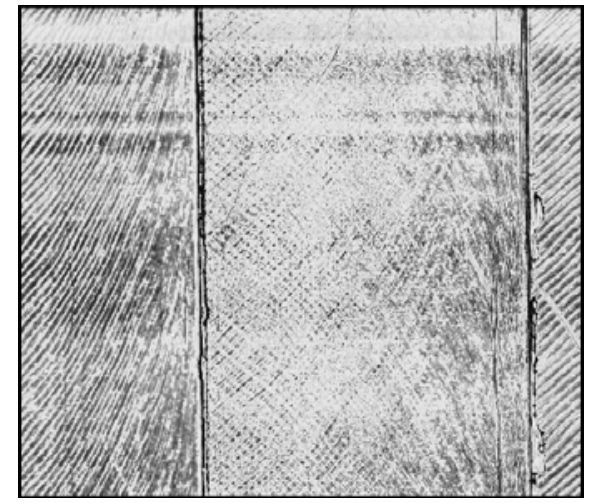

a)

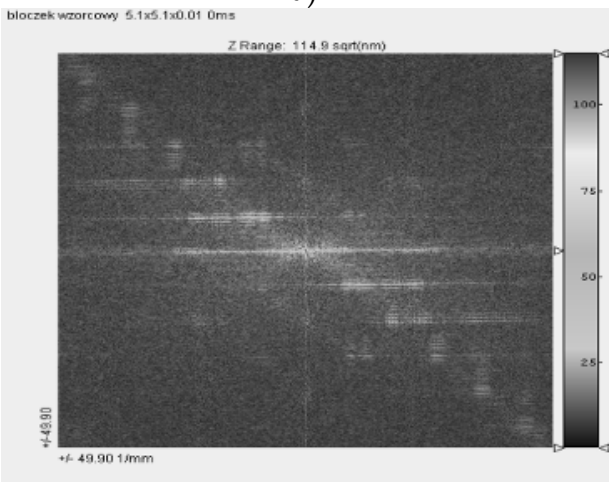

b)

Fig. 13. Surface profile -a and its FT -b of mechanically polished steel SWM7

Grooves and scratches visible in Fig. 13a are the result of the polishing process. These features cover the whole surface in a more or less uniform way and overlap. FFT allows toassess the repeatability of the processes forming the periodic inequalities. As may be noticed, in its two-dimensional FFT (Fig 13b) periodic dependence of the PSD along the direction forming an angle $45^{\circ}$ with the $X$ and $Y$ axes may be distinguished. It is result of interference of spatial waves appearing in $X$ and $Y$ directions. The occurrence of spatial waves interferences indicate the same processes along mutually perpendicular directions.

Fig. 14a shows FFT of optical profile of polyvinylcarbazole (PVK) film deposited on Corning glass 7059, obtained by spin -coating method [Patel et al. 2007], The scanning area was $10 \times 10 \mathrm{~mm}$ which enables detecting of surface features in longer space wavelengths. 
a)
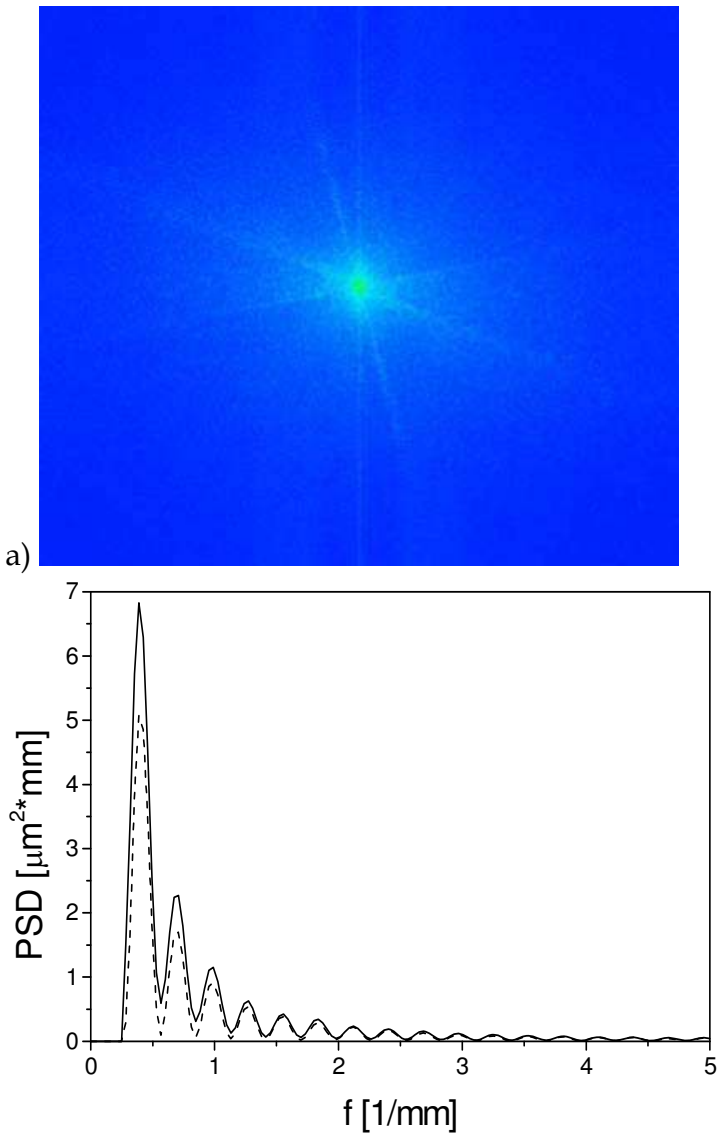

b)

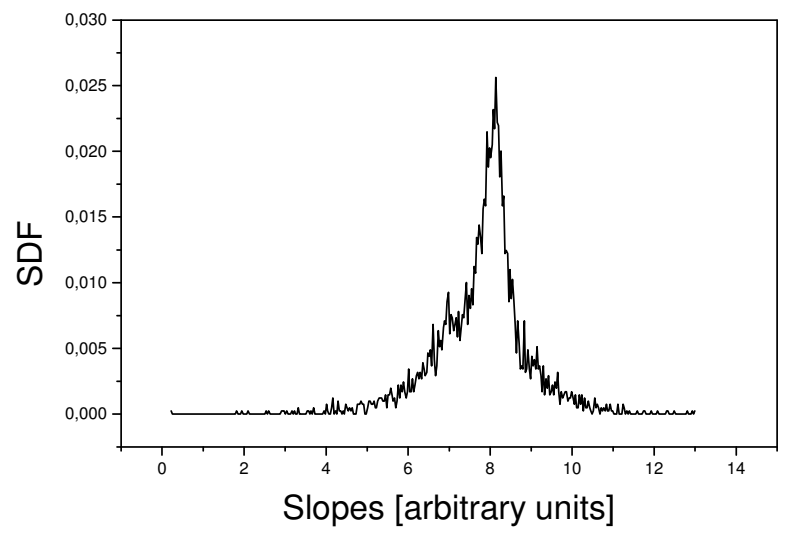

Fig. 14. FFTprofile of PVK film on Si substrate obtained by means OP BRDF mode -a and one dimensional PSD along $X$ and $Y$ axis $-b$, relative slopes distribution function for PVK film -c respectively 
Because refractive index of PVK for $633 \mathrm{~nm}$ light wavelength is nearly the same as Corning 7059 glass [10], almost the whole scattered light originates from upper surface of the film. FFT analysis of optical profiles shown in spatial frequencies, allows to distinguish periodic features and specify interference of spatial waves and to evaluate ratio of surface anisotropy. Because PVK was formed by centrifugal force in spin-coating process the PSD functions obtained for $X$ and $Y$ axes are similar, what is presented in Fig 14b. The determined PSD demonstrates as well periodic structure of surface. Also the relative slope density function (SDF) was determined for this sample (Fig. 14c).

OP measures the local slope of microfacets (local surfaces), the autocorrelation function is given as:

$$
A C F(s)=\sigma^{2} p(s)
$$

This relation may be applied to ACF determination directly from OP measurements if $p(s)$ is known.

Fig. 15a shows FT from optical profile of satinated glass texture used in glass houses. In Fig. $15 \mathrm{~b}$ the calculated PSD function for $\mathrm{x}$ and $\mathrm{y}$ spatial frequency is presented.
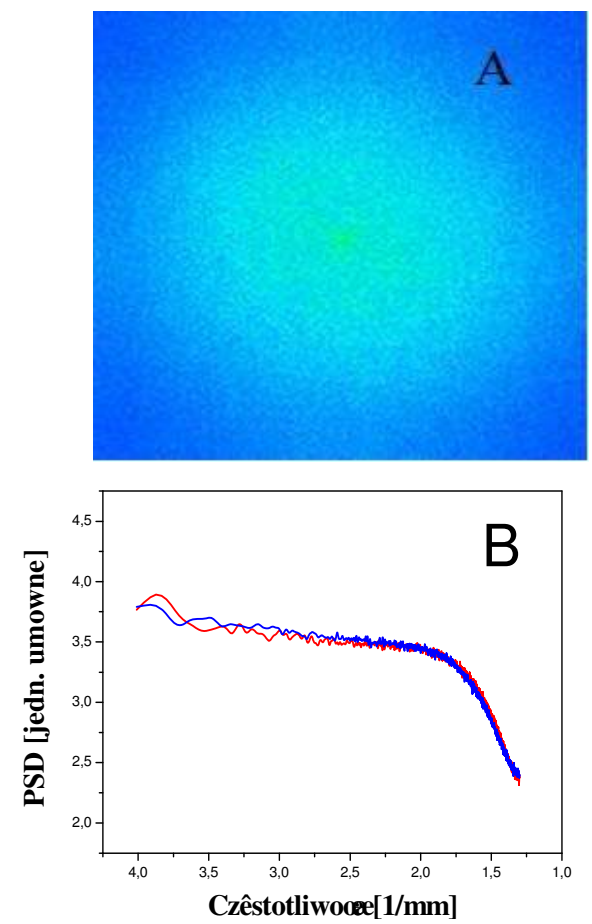

Fig. 15. FT of satinated glass surface and arbitrary one-dimensional PSD for $\mathrm{x}$ and $\mathrm{y}$ direction

As results from Fig 15.b the PSD for $\mathrm{x}$ and $\mathrm{y}$ directions are nearly the same. Thus one may be conclude that satinated glass surface exhibits very good isotropy. It is important factor for glass use in green houses. 
On the other hand the OP measurements allow to detect anisotropy of surface creation process. Optical profilometry could be applied to waviness detection occurring on surface or to inspection of periodic variation of film thickness. As an example in Fig. 16a the profilometric map of TiO2-SiO2 film on BK7 glass substrate obtained by dip coating sol-gel method is shown.

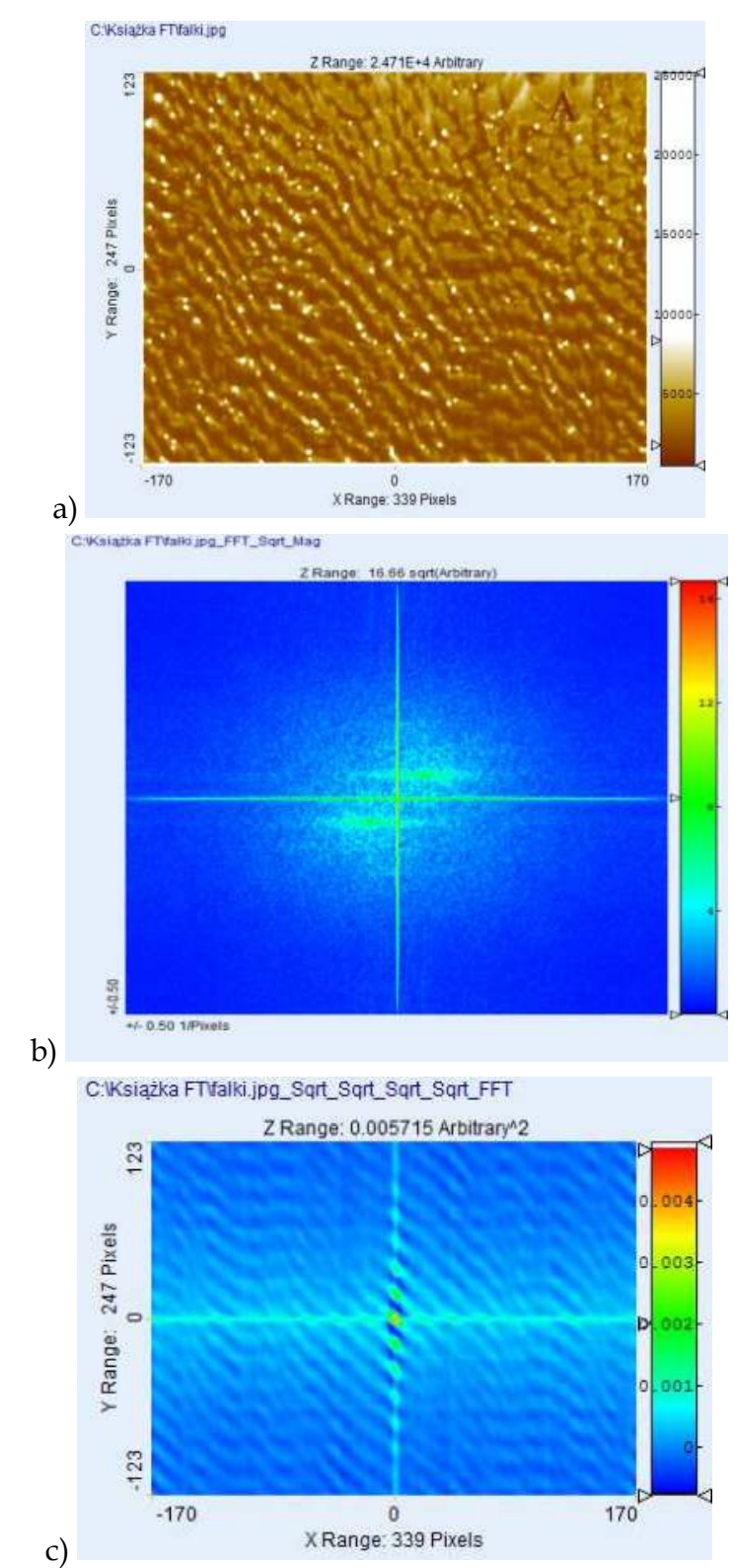

Fig. 16. PO profile of TiO2-SiO2 film on $-\mathrm{a}$ and its FFT - b and ACF -c 
It presents periodic changes of reflection coefficient for $\mathrm{TiO} 2-\mathrm{SiO} 2 \mathrm{film}$. In Fig. 16b the Fourier Transform of OP profile from Fig. 16a is shown. This is due to two harmonic vibrations caused by mechanical elements of technological equipment Elimination of vibration allows to obtain very flat $\mathrm{TiO} 2-\mathrm{SiO} 2$ films [Karasiński 2005].

\section{Conclusion}

As it is shown in this chapter the optical methods may be used as effective tools for surfaces and interfaces thin films and layers. Surface topographic parameters may be obtained from biredirectional reflectance distribution function and optical profilometry measurements. The BRDF and OP techniques allow to get information about surface topography. From these optical investigations the surface power spectral density (PSD) and autocorrelation (ACF) functions for surfaces and films may be found. Both functions are calculated from the Fourier transformation of surface profiles. The root mean square (rms) surface roughness, slopes and correlation length for studied samples from PSD function have may be determined.

The resolution of BRDF method allows to determine surface parameters in spatial wavelength range from 0.1 to hundreds of micrometers. The OP may be applied as complementary method for measurements of longer spatial wavelengths.

The most effective analysis of the surface statistic is calculating power spectral density or autocorrelation functions from surface profiles in spatial frequency or wavelength domains respectively.

The scattered radiation is a function of highs of irregularities and slopes of microfacets but the sensitivity of $\mathrm{PO}$ studies is resulted mainly from sensitivity of detection of slopes change. In PO BRDF mode the technique is sensitive for upper surface of a film when substrate is optically flat. The presented techniques may be also take advantage in control manual painting or varnishing. The presence of long lateral irregularities is often caused by manual or mechanical treatments (i.e paintings, varnishing, manual polishing) and may have periodical nature. The short spatial waves result rather from random process of surface formation and their contribution in total profile is easy to determine from light scattering by the using BRDF method.

If films are transparent and their interfaces are partly correlated, the description of film topography is difficult.

The PO specular mode may be used for transparent film thickness determination if upper and lower interfaces are flat. This technique allows measures thickness of layer If interfaces correlated are in transparent films. If surface are partially correlated the BRDF -PO mode is more preferred for their topography measurements

\section{Acknowledgment}

This work was supported by Grant N N503 137235 of Polish Science Committee.

\section{References}

\section{Print Books}

Stover J.C., Optical Scattering. (1995). Measurements and Analysis (2d ed.), SPIE, ISBN 0-81941934-6, Belligham. (b)

Bennett J. M.,. Mattsson L. (1999). Introduction to surface roughness and Scattering (2 ed.) SA ISBN 1-55752-609-5, Washington, D.C. 
Workmann J. ( st ed.) (1998). Applied spectroscopy, Academic Press, ISBN 0-112-764070-3.

Whitehouse D. J. (2003). Handbook of surface and nanometrology, (1st-ed.) IOP Publishing, ISBN 0-7503-0583-5, Bristol and Philadelphia.

Beckmann P, Spizinochino B. (1963) The scattering of electromagnetics waves from rough surfaces, Pergamon Press Oxford, London, New York, Paris:

\section{Papers in Journals}

Elson J.M., Bennett J.M., (1979). Vector scattering theory, Opt. Eng., 18, 116.

Church E.L., Jenkinson H.A.,. Zavada J.M, (1977). Measurement of the finish of diamondturned metal surfaces by differential light scattering, Opt. Eng., 16360.

Chandley P.J. (1976). Determination of the autocorrelation function of height on rough surface from coherent light scattering, Opt. Quant. Elect. 8329.

Church E. L. (1988). Fractal surface finish, Appl. Opt. 271518.

Ohlidal I., (1988). General formulas for the optical characterization of single layers with spectroscopic reflectometry, J. Mod. Opt. 35, 1373.

Stover J.C., Serati S.A., Gillespie C.H., (1984). Calculation of surface statistic from light scattering, Opt. Engng, $23,406$.

Karasiński P. (2005) Influence of technological parameters on the properties of sol-gel silica films, Opt. Appl. Vol. 35, No. 1117.

ANSI/ASME (1985). Standard B46.1-1985, Surface Texture (Surface Roughness, Waviness, and Lay), Am. Soc. Mech. Eng., New York.

Elson J. M. (1984). Theory of light scattering form rough surface with inhomogeneous dielectric permittivity. Phys. Rev. B30 5460.

Elson J. M. Benett J. M. (1995) Calculation of the power spectral density from surface profile data, Appl. Opt., 34, 20.

Stover J.C., Serati S.A., Gillespie C.H., (1984).Calculation of surface statistic from light scattering, Opt. Engng, 23406.

Bendat, J.S., Piersol, A.G. (1971) Random Data: Analysis and Measurement, Proceedures, WileyInterscience, New York

Bendat, J.S., Piersol, A.G. (1986) Engineering Applications of Correlation and Spectral Analysis, ( 2 d ed.), John Willey \&Sons, New York.

Nicodemus F. E. (1965). Directional Reflectance and Emissivity of an Opaque Surface Applied Optics, 4767.

Mainsah E., Dong W.P., Stout K.J., (1996) .Measurement 17173.

\section{Papers in Conference Proceedings}

Kaczmar J.W., Pietrzak K., Wlosinski W. (2000) The production and application of metal matrix composite materials. J Mater Process Technol., 10658.

Karasiński P. (2005). Influence of technological parameters on the properties of sol-gel silica films, Opt. Appl. 35, 117

M.J. Elson, (1992) Theory and Software for Light Scattering from Multilayer Optical Components with Interfacial Roughness, Naval Air Warfare Center, Weapons Division, China Lake, CA, Technical Publication 8084.

Stover J.C. (ed.), (1995). Optical Scattering in the Optics, Semiconductor and Computer Disk Industries, Proc. SPIE 2541. (a)

Patel S., Ramrakhiani M., Bisen D., P. (2007) J. Appl. Polym. Sci, 104, 722. 
Church E. L., Leonard T.A., Takacs P. Z. (1989). The prediction of BRDF from surface profile measurements. Proc. SPIE 1165196.

Brown A.J.C., Breitmeier U., (1988) Industrial application of an optical profilometer. Proceedings SPIE, 954.

Lipiński M., Cichoszewski J. (2008) in Proc. 23nd EU PVSEC, (eds. G. Willeke, H. Ossenbrink, and P. Helm, WIPRenewable Energies, Munich, Germany, 1911. 


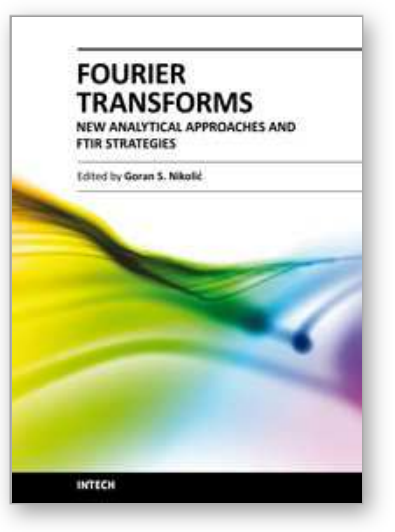

\author{
Fourier Transforms - New Analytical Approaches and FTIR \\ Strategies \\ Edited by Prof. Goran Nikolic
}

ISBN 978-953-307-232-6

Hard cover, 520 pages

Publisher InTech

Published online 01, April, 2011

Published in print edition April, 2011

New analytical strategies and techniques are necessary to meet requirements of modern technologies and new materials. In this sense, this book provides a thorough review of current analytical approaches, industrial practices, and strategies in Fourier transform application.

\title{
How to reference
}

In order to correctly reference this scholarly work, feel free to copy and paste the following:

Janusz Jaglarz (2011). Description of Topography of Surfaces and Thin Films with the use Fourier Transformation, Obtained from Non-Standard Optical Measurements, Fourier Transforms - New Analytical Approaches and FTIR Strategies, Prof. Goran Nikolic (Ed.), ISBN: 978-953-307-232-6, InTech, Available from: http://www.intechopen.com/books/fourier-transforms-new-analytical-approaches-and-ftirstrategies/description-of-topography-of-surfaces-and-thin-films-with-the-use-fourier-transformation-obtained-fr

\section{INTECH}

open science | open minds

\author{
InTech Europe \\ University Campus STeP Ri \\ Slavka Krautzeka 83/A \\ 51000 Rijeka, Croatia \\ Phone: +385 (51) 770447 \\ Fax: +385 (51) 686166 \\ www.intechopen.com
}

\author{
InTech China \\ Unit 405, Office Block, Hotel Equatorial Shanghai \\ No.65, Yan An Road (West), Shanghai, 200040, China \\ 中国上海市延安西路65号上海国际贵都大饭店办公楼 405 单元 \\ Phone: +86-21-62489820 \\ Fax: $+86-21-62489821$
}


(C) 2011 The Author(s). Licensee IntechOpen. This chapter is distributed under the terms of the Creative Commons Attribution-NonCommercialShareAlike-3.0 License, which permits use, distribution and reproduction for non-commercial purposes, provided the original is properly cited and derivative works building on this content are distributed under the same license. 